\title{
Web-Services-based Architecture for Information Integration in Nigeria Deregulated Electricity Market Environment
}

\author{
Joseph O. Dada \\ School of Computer Science \\ Manchester Institute of Biotechnology \\ Faculty of Engineering and Physical Sciences \\ University of Manchester, UK
}

\begin{abstract}
The recent handling over of the assets of Power Holding Company of Nigeria (PHCN) successor companies to the core investors was a major landmark in the reform of the electric power sector in Nigeria. However, the emergence of the different autonomous utilities for running the deregulated electricity market has great consequent for the day-to-day planning and operation of the power grid. There is need to exchange information and data between applications of the entities not only to guarantee the security and efficient operation of the power grid, but also for settlement purposes. This paper proposes a Web-services-based architecture for information integration between applications of the autonomous entities in deregulated electricity market. Loosely coupled feature of Web services provides more flexible and reliable way to integrate information in heterogeneous environments resulting from the unbundled vertically integrated national grid in Nigeria.
\end{abstract}

\section{General Terms:}

Information Exchange, Heterogeneous IT Environments, Loosely Coupled Systems

\section{Keywords:}

Deregulated Electricity Market, Information Integration, ServiceOriented Architecture, Web Services, Data Exchange

\section{INTRODUCTION}

The Nigeria Electric Power Sector Reform Act (EPSRA) of 2005 [1] has finally transformed the vertically integrated utility - PHCN into different entities in readiness for private participation in the operation of competitive electricity market in Nigeria [2]. The recent handling over of assets of the PHCN successor companies to the private core investors mark the beginning of competition in electricity in the country. This will lead to major changes in the organizational structures of the electricity supply industry as well as the operation of power systems [3] in the country.

The separation of natural monopoly power network grid from the competitive elements has a great effect not only on the market op- erations, but also on many areas such as planning, operation and maintenance of the power systems that were traditionally carried out by vertically integrated utility. Furthermore, electric energy is not a simple commodity. Unlike other forms of energy, it cannot easily be stored in large quantities. The handling of the new business processes and the data interchange between the complex assembly of electricity players therefore require a good information integration mechanism. The integration mechanism must not only consider the geographically distributed organizations (hereafter referred to as actors) concerned with the electricity network and market operation, but also the heterogeneous nature of the IT's environments and applications, including legacy application systems for the autonomous entities [4, 5] 6].

The need for automation of data exchange and information infrastructures for the new deregulated electricity market in Nigeria has been emphasized in [6] where XML-based information exchange framework was presented, and [2] that proposed a conceptual model of information exchange network using object-oriented approach. However, technique and communication protocol for automation of data exchange and information integration between applications within and outside organization boundaries are not yet addressed. There are a numbers of potential candidates that can be used. For example, one could use Common Object Request Broker Architecture (CORBA), Java Remote Method Invocation (RMI) or Distributed Component Object Model (DCOM) for the integration and data exchange, but these technologies require a common security domain context, function calling convention, binary data types, and way of locating and activating remote applications. They are better suited to assembly of tightly coupled components, and less suitable to loosely coupled systems, which only need to exchange information and data $[5]$.

A better approach for integration of loosely coupled systems is the Web Services implementation of Service-Oriented Architecture (SOA). Web services allow seamless applications integration by making it easier to tie applications running on heterogeneous platforms together helping them communicate correctly, effectively and at reduced cost $|7|$.

Significant research efforts have been carried out on the application of SOA and Web services for information and data integra- 
tion in the electric power system industry, among which are Webservices infrastructure for information integration in power systems [3], Web-services-based framework for integration of power system applications [8], a distributed service-oriented control infrastructure for smart grid [9] and Web service workflow for power system security assessment [10]. However, there is presently no application of these to the information integration for the newly created organizations that will be responsible for the operation of deregulated electricity market in Nigeria. This work proposes Web Services architecture for an open, flexible, and scalable information integration infrastructure with higher cooperation and integration capability for different applications and actors in the competitive electricity market environment in Nigeria. The proposed Web Services architecture is platform, language, and vendor independent, and thus it is an ideal candidate for an effective integration of new and legacy applications. This will provide a low-cost and high-performance information integration allowing easy cooperation between big as well as small companies in Nigeria deregulated electricity market leading to an effective management of the security and reliability of the grid network. Service-oriented infrastructure fulfills the shortand long-term information integration needs and will facilitate frequent changes of actors' applications and business partners in the evolving electricity market in Nigeria.

The rest of the article is organized as follows. Section 2 briefly describes the deregulated electricity market in Nigeria together with emphasis on the market structure and applications for enabling network and market operations. In section 3, Service-Oriented Architecture (SOA) and Web services technology are discussed. Section 4 presents the architecture of the Web services information integration for the Nigeria electricity market and the article ends with conclusions in section 5 .

\section{NIGERIA DEREGULATED ELECTRICITY MARKET}

The Nigeria Electric Power Sector Reform Act (EPSRA) of 2005 provides the legal framework for the separation of vertically integrated Nigeria power utility - PHCN - into generation, transmission and distribution companies. The Act stipulates the functional unbundling of the generation, transmission and distribution sectors. The unbundling of PHCN has resulted into creation of 18 successor companies, including 6 generation companies, a transmission company and 11 distribution companies (Discos). EPSRA provides an enabling regulatory framework for private sector participation in the electricity industry in Nigeria. The Act also provides the establishment of a regulatory body - National Electric Reforms Commission (NERC) to facilitate the privatization of the successor companies as well as the gradual development of a competitive and privately managed electricity sector [6]. In addition to the generation companies, a number of privately financed Independent Power Projects (IPPs) are emerging and many others have been licensed by the NERC [11] (hereafter the new created 6 generation companies and IPPs are referred to as Gencos). The rest of this section briefly describes the electricity market structure and the functions of the actors including applications for performing their functions in the deregulated electricity market.

\subsection{Electricity Market Structure}

A good market structure is very crucial to a successful take of a newly deregulated electricity market. Nigeria electricity market is structured to follow a single buyer model initially during the transitional period. In this model, the Bulk Trader (Single Buyer) is responsible for buying electricity generation from the Gencos and resell same to the Discos that are responsible for supplying the consumers in their respective geographical zone. Single buyer model is a transitional arrangement to assure the Gencos that they will be paid for power produced and supplied to the Discos. This arrangement effectively shift the risks involved in electricity trading to the single buyer to allow the market to mature until Discos are capable of standing alone. Wholesale competition model that allows direct electricity trading between the Gencos and Discos is expected to take over from the single buyer model after transitional electricity market arrangement.

The adopted market structure requires various actors including Market Operator (MO), Gencos, Transmission Company of Nigeria (TCN) or System Operator (SO), Discos and the Nigeria Bulk Electricity Trader (NBET). The actors require diverse set of applications for the daily execution of the electricity business processes in the new electricity market. Some of the applications needed by each actor for fulfilling their functions and business processes as required by the Grid Code [12], Distribution Code [13] and Market Rules [14] are identified below:

MO:

-Market Settlement System (MSS)

- Contract Registration System (CRS)

-Imbalance Quantity Calculation (IQC)

- Meter Data Management (MDM)

-Dispatch Management (DM)

-Ancillary Service Management (ASM)

-Data Storage System (DSS)

Genco:

-Power Purchase Management (PPMS)

-Nomination Management System (NMS)

-Contract Management System (CMS)

-Automatic Generation Control (AGC)

-Ancillary Services Contract (ASC)

-Distribution Management System (DMS)

—Data Storage System (DSS)

Disco:

—Automated Meter Reading (AMR)

- Meter Data Management (MDM)

-Outage Management System (OMS)

-Load Forecasting (LF)

-Power Purchase Management (PPM)

-Customer Information Systems (CIS)

—Data Storage System (DSS)

TCN/SO:

-Energy Management System (EMS)

- Transmission Usage Management (TUM)

—Load Data Management (LDM)

- Load Flow Analysis (LFA

-Congestion Management System (CMS)

—Data Storage System (DSS)

NBET:

-Power Purchase Management System (PPMS)

- Contract Management System (CMS)

-Bilateral Transaction (BT)

-Data Storage System (DSS) 


\section{SERVICE-ORIENTED ARCHITECTURE AND WEB SERVICES}

Here we introduce the concepts of SOA and Web services used for the development of information integration for the applications in the electricity market.

\subsection{Service-Orientation}

Service-orientation introduces new concepts that augment the manner in which enterprise logic (e.g. enterprise logic for individual actor described in section 2 above) is represented, viewed, modeled, and shared. The software engineering theory of separation of concerns that promote the breakdown of a large problem into a series of individual concerns is believed to have inspired serviceorientation. The theory allows the logic required to solve a problem to be decomposed into a collection of smaller, related pieces. Each piece of logic addresses a specific concern [15]. Messages, operations, services and processes are the main concepts in ServiceOriented Architecture (SOA). A service consists of a set of operations and communicates with other services or applications through exchange of messages. A service encapsulates specific application logic by exposing its functionality through a well-defined and language-independent interface.

Services can be loosely coupled to create flexible dynamic business processes and agile applications that may span several organizations and computing platforms [16]. The service provider encapsulates its service implementations and describes service capabilities, interfaces as well as qualities. The descriptions are published to the outside world where these services can be discovered, selected, and bound by service consumers [17]. SOA provides a distributed computing infrastructure for both intra- and cross-enterprise application integration and collaboration [16].

Web Services technology is one of the many ways to implement SOA. Other ways are common object request broker architecture (CORBA) and message-oriented middleware systems. However, these are tightly coupled protocols and very limited in terms of flexibility and re-usability compared to Web services. Web service technologies are particularly suitable for information integration due to their ease of application programming, loose coupling, portability, maintenance, and integration with legacy services [18].

\subsection{Web Services}

Web service is a widely used standard for interoperable machineto-machine interaction over a network. Web services follow standard protocols approved by the W3C organization. A Web service is a system implemented by a software agent capable of sending and receiving messages defined through the Web services description language (WSDL, an XML-based language), and passed through the HTTP protocol. WSDL makes possible the discovery of Web services through service brokers [19]. The basic architecture of a Web service (Fig. 1) consists of a service provider, a service consumer and a service registry. A Web service provider publishes its service interface (WSDL) in the registry through which a Web service consumer discovers it using Universal Discovery, Directory and Integration (UDDI) protocol. A discovered service is then bind to consumer's application or another Web service using Simple Object Access Protocol (SOAP), a standard XML-based communication protocol for messaging over the Internet.

A new or existing application can be exposed through a SOAP interface to allow it to be accessed by other applications or Web ser-

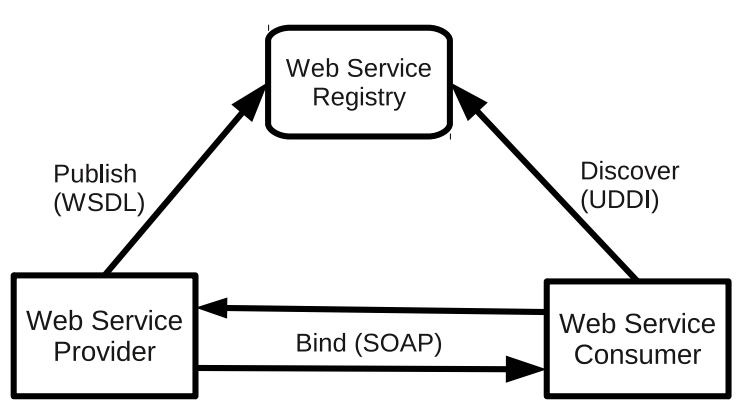

Fig. 1. Web-Services Basic Architecture.

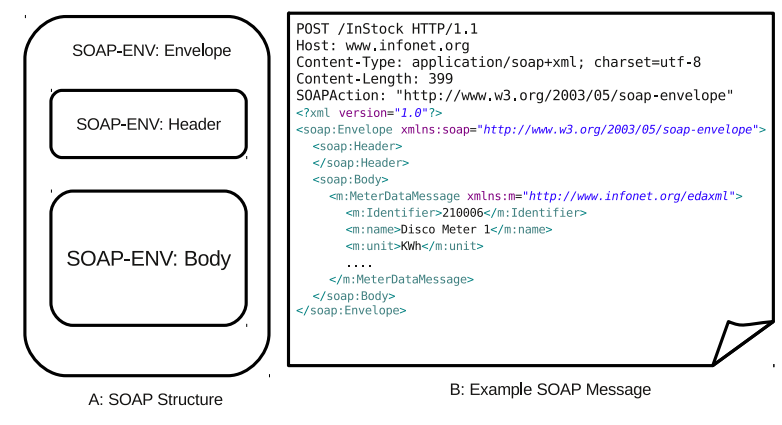

Fig. 2. Structure of SOAP message and SOAP message. example.

vices over the Internet. The structure of a SOAP message from a Web service consists of XML envelope element used to wrap the user-defined messages. The SOAP structure and an example SOAP message are illustrated in Fig. 2.

The use of standard XML protocols makes Web service technologies platform, language, and vendor independent; thus they are an ideal candidate for use in the integration of legacy power system and new emerging applications for the electricity utilities [18].

\section{SERVICE-ORIENTED INFORMATION INTEGRATION}

The performance of various business processes in Nigeria deregulated electricity market requires the applications for actors described above to be integrated together. The integration can be achieved through function integration and data exchange. Function integration involves one application programmatically invoking the code that lies in another application, while the data exchange mechanism uses tools to move data from one application to the others [8]. Applications integration without a proper integration framework in place will lead to complex point-to-point linking of intraand inter-organization applications requiring tying of an application to multiple interfaces. This situation can lead to large multitude of dependencies as the number of the electricity market actors with diverse applications increases. A better way to achieve integration is by exposing the application's data and functionality in a language-independent manner using Web services. This section presents the Web-services-based information integration architecture for the deregulated electricity market to help overcome these integration issues. 


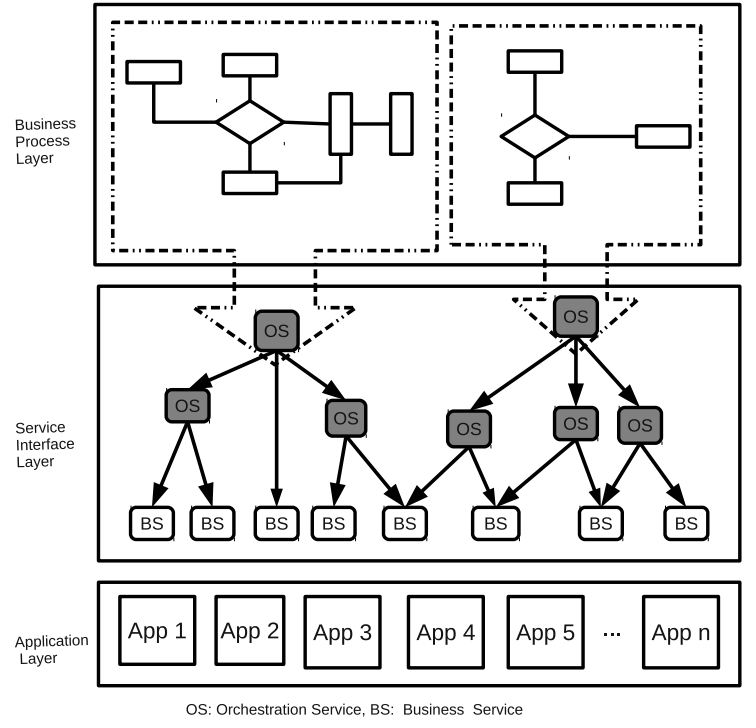

Fig. 3. Services integration using service orchestration approach.

\subsection{Integration of Services using Orchestration Approach}

Applying the concept of service-orientation to the electricity market requires the applications identified in section 2 to be exposed as service interfaces (service interfaces are hereafter referred to as services). These services can be implemented using Web services framework. Mapping each application directly to a Web service or multiple Web services will however result in many Web services that need to interact with one another for execution of business processes. Web-services-based function integration involves calling the functions in other applications through SOAP messaging [8]. This integration approach although better than application-toapplication integration will still lead to many point-to-point links between the participating intra- and inter-organization Web services because each Web service has to know the interfaces of the Web services that it calls. Composing and aggregating the atomic business services into composite services in a well-organized manner using service orchestration approach can help overcome this problem. Using this approach the integration procedures can be separated into a decoupled layer that implements electricity business logic [15].

An orchestration service controls the step-by-step actions of integration, moving the integration process from one state to another. At each step, it calls a participating service and then moves on to the next step, which may require the use of a different service. The orchestration service unifies its participating business functions and exposes them through a single entry point, therefore simplifying external communication. The composition or aggregation of higherlevel orchestration service can also involve other orchestration services as illustrated in Fig. 3. Using orchestration service to control the interaction of business functions and to provide one central service significantly enhances the manageability and maintainability within an integrated system [8].

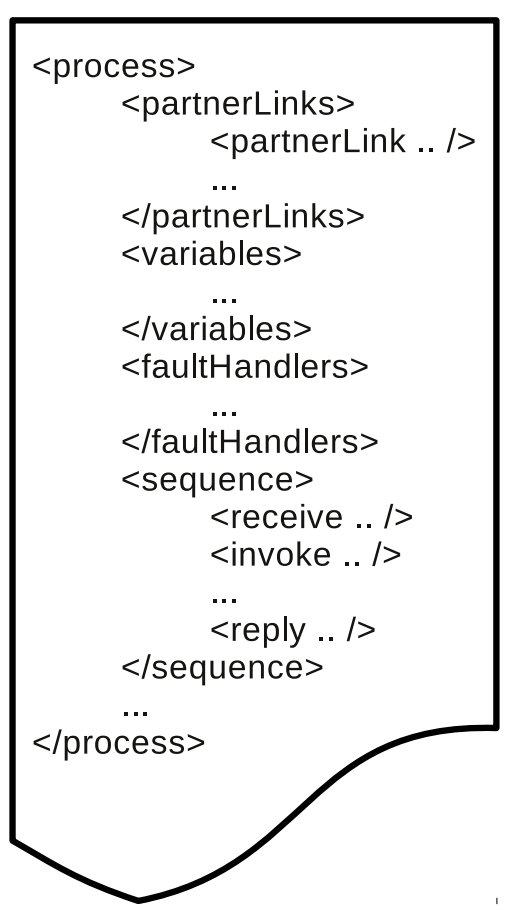

Fig. 4. Structure of WS-BPEL process definition.

\subsection{Business Process Execution Language}

The Web services solve the interoperability problems of heterogeneous applications, but they are still not sufficient for large-scale industrial applications because of the lack of abilities to construct intra- and inter-organization orchestration systems. To address this, several languages, such as ebXML, and WS-BPEL [20], OWL and OWL-S to describe orchestration of business processes have been proposed on top of those basic business services for automating business process management. Among these languages, WS-BPEL is particularly notable for providing a formal specification of business processes and business interaction protocols.

WS-BPEL uses executable business process and abstract process to ensure that different business processes can understand one another in a Web services environment [21]. It is gradually becoming a defacto standard for Web services orchestration. WS-BPEL process definition involves the description of interaction between partners. A process can interact synchronously or asynchronously with its partners, i.e. its clients and with the services the process orchestrates. WS-BPEL supports both sequential and concurrent execution process activities. The building blocks for a BPEL process are the descriptions of the parties participating in the process, the data that flows through the process and the activities performed during the execution of the process. WS-BPEL processes can be executed via their own Web service interface, or through internal triggers defined inside the process [22]. The basic structure of process definition in WS-BPEL is shown in Fig. 4.

Many orchestration engines for executing the business processes encoded in WS-BPEL standard are available. Here we propose the use of popular Apache Orchestration Director Engine (ODE) 
for Nigeria electricity market. It is open source software and well documented. ODE software executes business processes written in WS-BPEL standard. It talks to Web services, sending and receiving messages, handling data manipulation and error recovery as described by the process definition [23].

\subsection{Data Exchange Format for Deregulated Electricity Market}

Service orchestration approach as described above provides effective way to composed atomic services into high-level services for information integration. This alone is not enough to meet the requirement of data exchange between electricity actors in the deregulated market. Inter-enterprise data exchange requires common data model and agreed standardized exchanging format to prevent the development of multiple message models for several services. Seamless integration of market actors' proprietary information requires a standard way for describing the electricity market resources, their attributes, and relationships.

The important role of information exchange in electric power network operations led to the adoption of Common Information Model (CIM) as standard to exchange and manage network models by many electricity utilities all over the world [24, 25, 26, 27, 28]. There are also efforts to extend CIM to support electricity market communications [29 30]. While the CIM standard can be adapted to cater for the exchange of network models in Nigeria electricity market environment, the information model for the market operations needs to be tailored towards the adopted market model in Nigeria as defined in Market Rules and Grid Code [2].

A first attempt to developed common data model for information exchange in Nigeria electricity market has been made in [6] where the development of data model based on common business process interaction patterns was proposed. This idea was further developed in [2] by creating a first data exchange formal in XMLbased standard called Electricity DAta eXchange Markup Language (EDAXML) for electricity market in Nigeria.

The EDAXML model was based on object-oriented model of the business process interaction patterns. Since the Web services exchange information in XML, a universal format for structured documents, EDAXML is a good candidate for defining messages for the higher-level services responsible for inter-enterprise information integration in Nigeria electricity market. A WSDL document that is generated from the EDAXML model basically defines Web service requests and responses in terms of the messages that are defined in EDAXML model. A variety of specification and standards, such as eXtensible Stylesheet Language (XSL), Document Object Model (DOM) and Simple API for XML (SAX) for XML document processing making it easy for the sending and receiving applications to process it. Documents based on this agreed XMLbased format can be readily parsed and consumed by heterogeneous applications belonging to two actors as illustrated in Fig. 5 .

\subsection{Design of Web Services Architecture}

Designing the Web services information integration architecture involves the designing of the basic atomic Web services and the business processes using the concepts described above. This section describes how the applications of the actors are exposed as Web services as well as their integration into architecture for intra- and inter- organization applications integration.

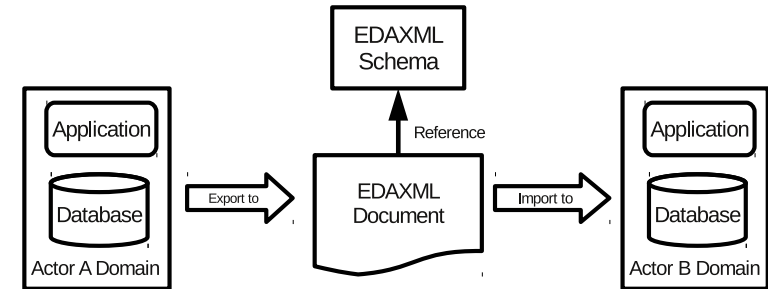

Fig. 5. Data exchange using EDAXML.

4.4.1 Design of Web Services. Identification of the right granularity for the services is an important issue to be resolved while building Web services. For this type of evolving electricity market, it is natural to choose the granularity that follows the individual application for performing specific function by the organizations. As discussed in section 2, five main classes of actors will be actively involved in the electricity market transactions in Nigeria. Each actor in each of the classes will be an autonomous entity operating their own applications and tools for the fulfillment of its roles and functions in the electricity market. We therefore aligned the granularity for the services to the application for performing the specific function. The development of the Web service can be messageoriented or RPC based Web services. Message-oriented approach is proposed because of its suitability for integrating loosely coupled systems.

The next issue is the choice of Web service interface development approach. Most of the efforts on this are spent on the service operations. Two main approaches can be adopted: a top-down or bottomup approach. To avoid interoperability problems and to conform to Web Services Interoperability (WS-I) basic profile [31], top-down approach is usually preferred. Basically this starts with the definition of the WSDL. A stub code for the service is generated automatically and the developer then completes the implementation of the Web service. De-coupling service implementation logic from the service interface definition permits the individual actor to develop its services separately and independent of other services' internal implementation.

4.4.2 Design of Business Processes. Several intra- and interorganization business processes are required for the electricity market operations. Designing these processes involves the composition or aggregation of the atomic business services for the applications into composite services. An example of such business processes is the submission of meter data by a Discos to the MO. The submission of meter data business process will normally involve the reading of meters, normalization of the meter data, storing the meter data in database, creation of the meter data message, and eventual sending of the meter data message to the MO, who in turn process the message and send an acknowledgment back to the Disco. This clearly shows that many basic business services and orchestration services both at the MO and Disco domains are required for the completion of meter data submission process.

The interactions of the services are based on the exchange of messages. The sender of a message opens a connection to a receiving port, posts the request message, and receives an acknowledgment possibly combined with a response body resulting from the processing of the request message. Messages must be self-sufficient, containing or referencing all of the information necessary to understand the contents of the message [8]. Fig. 6 and Fig. 7 show 


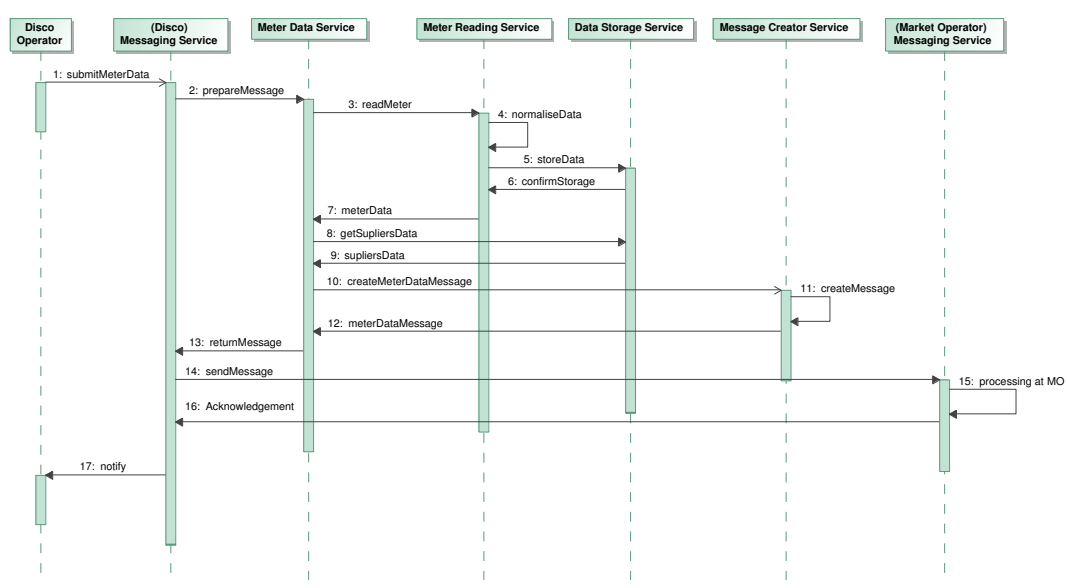

Fig. 6. Submission of meter data process at the Disco side.

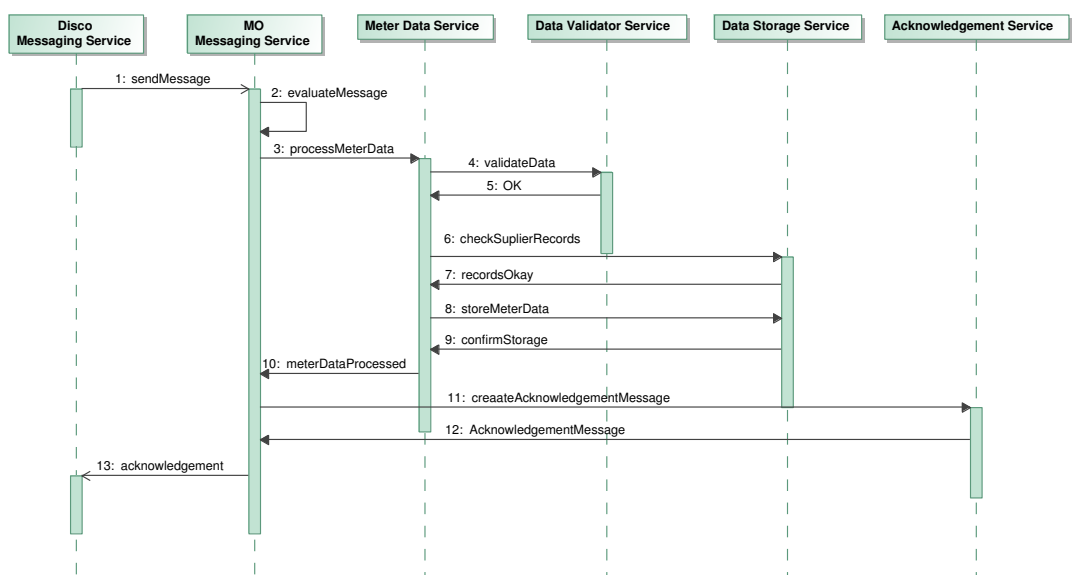

Fig. 7. Meter data submission process at the MO side.

the sequence diagrams illustrating how the atomic business and orchestration services interact by sending and receiving messages to fulfill the meter data submission process. A very important orchestration service involved in the meter data submission process is the Messaging Service (MS). MS resides in each actor's domain and is responsible not only for coordinating the meter data message submission process between the $\mathrm{MO}$ and Disco, but also for all inter-actors exchange of messages. MS in each actor's domain is expected to understand all the messages it is required to send and receive in accordance with the roles and functions of its actor in electricity market as defined earlier.

\subsection{Web-Services-based Architecture}

We developed a Web-services-based architecture for Nigeria deregulated electricity market environment using the generic framework developed above. Fig. 8 shows an example Web-services-based architecture for MO representing only its internal structure with MS serving as gateway for the exchange of information with other actors. The architectures of the other actors are similarly structured based on the applications for fulfilling their roles and functions in the electricity market. However, only MO architecture has the EDAXML repository and a single service registry residing at the
$\mathrm{MO}$ is also encouraged for all the actors. The overall Web-servicesbased architecture for the market is illustrated in Fig. 9 with emphasis on only information interchange between the actors using the MSs that communicate through the Internet.

A unique feature of this architecture is the easy with which services can be added or composed as the need arises. This is made possible by the orchestration service approach. Orchestration service provides the capability to add new services as they evolve. New actors can join the information integration system through the deployment of gateway orchestration service (the MS service) that described its relationships with other actors. SOA based system provides many benefits including improvement of interoperability and the integration of new and legacy applications, the ability to survive evolutionary changes in the structure, and implementation of the internals of each service [18]. This is especially important for Nigeria electricity market that is still evolving.

\subsection{Implementation Methodology}

The deregulated electricity market in Nigeria is recent and the operation of the market is due to commence soon. Some of the applications identified in section 2 will have to be newly developed, 


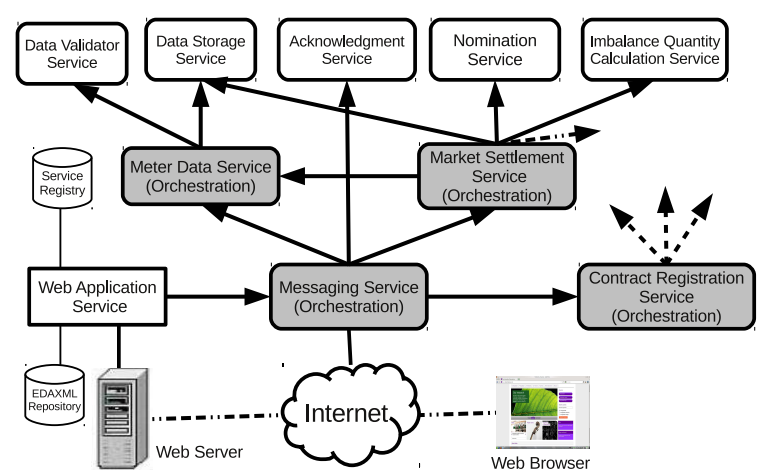

Fig. 8. Architecture of Web-services-based integration for MO applications.

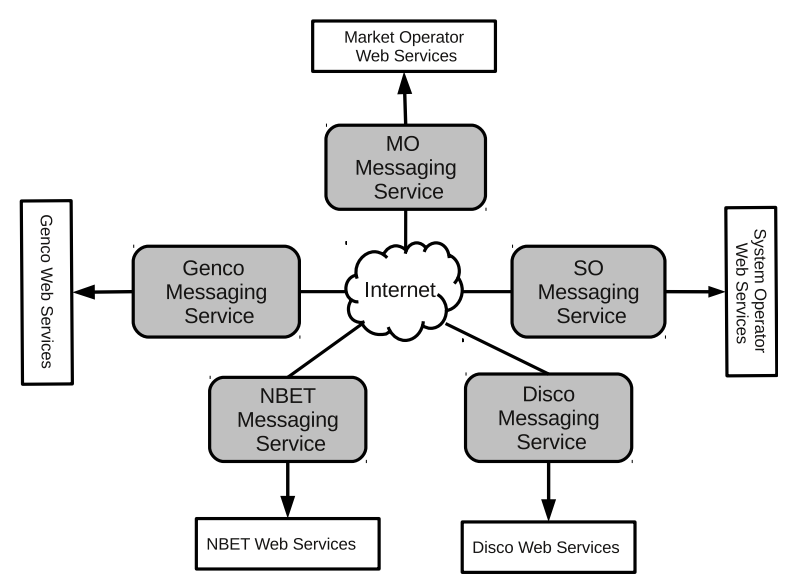

Fig. 9. Web-services-based architecture for inter-actors applications integration. There is one messaging (orchestration) service for each of the actors.

while others may be inherited from the assets of PHCN. A temporary method for data exchange is likely to be put place during the initial phase of the electricity market operations. During this phase, the process of gradual implementation and testing of the presented Web-services architecture can be started. Several software tools are available for constructing the WS-BPEL processes and for the development of WSDL for the Web services. Actual coding of the WSDL service interfaces can be carried out by the individual actors based on their preferred programming language, while processes defined in WS-BPEL using WSDL can directly be deployed into in a suitable orchestration engine. A typical implementation steps for MO architecture using the proposed Apache ODE for execution of WS-BPEL processes is depicted in Fig. 10. Here Java is the implementation language for WSDL services and Apache Tomcat serves as executing server for the Web services and ODE engine.

\section{CONCLUSIONS}

The privatization of the electric power sector in Nigeria has moved the country into an uncharted territory that requires a lot of responsibilities from all the actors in the electricity network and market operation in order to fulfill the objective of the liberalization of the

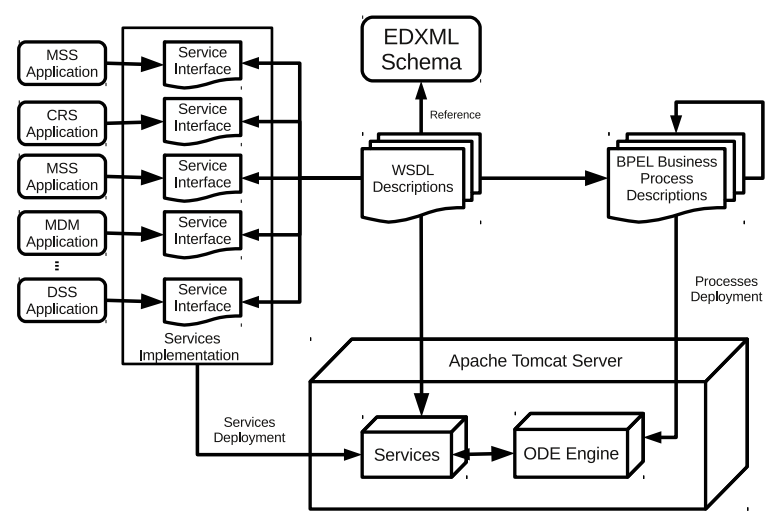

Fig. 10. Implementation steps for $\mathrm{MO}$ architecture.

electric power sector. Central to this is the information integration between the intra- and inter- organization applications. In this work, an architecture for Web services, which permits data and information integration has been developed and discussed. The architecture is aligned to the electricity market model and actors involved in the market and network operations.

A service orchestration approach for reducing the complexity in the applications integration is adopted to structure the intra- and inter- actor Web services. We adopted WS-BPEL for constructing the business processes for the orchestration services and Apache ODE for compilation and execution of the WS-BPEL orchestration processes. The architecture will enable automation of information and data exchange between the complex assembly of services and organizations involved in Nigeria liberalized electricity market with minimum human interactions. Using a loosely coupled set of services with a decoupled orchestration layer enables easy modification of the architecture to address the changing business needs of the evolving electricity market in Nigeria. The developed architecture shows and demonstrates the usefulness and suitability of Web Services and standard Internet based technologies for loosely coupled systems. Using Web Services paradigm overcomes the risk associated with tightly coupled middleware-based systems. SOA provides an easy way for interfacing and integrating the loosely geographically distributed applications of the actors together. This is especially important for the evolving electricity market that needs continuous incorporation of new actors into the information exchange infrastructures.

Future work will consist of using an agent-based approach to automatically construct and compile the WS-BPEL business processes. This will speed up the process of adding new services and actors into the system. Additionally, we would like to demonstrate the power of combining Web services by creating distributed workflows involving services for computational intensive applications.

\section{ACKNOWLEDGMENTS}

The author would like to thank his line manager (Pedro Mendes) and University of Manchester for the provision of resources use for this work.

\section{REFERENCES}

[1] Electricity-Act: Nigeria Electricity Reform Act. Technical report, Federal Republic of Nigeria, 2005. 
[2] J.O. Dada. Conceptual Modelling of Information Exchange Network for Nigeria Deregulated Electricity Market using Object-Oriented Approach. International Journal of Engineering and Technology, 3(4):449-463, 2013.

[3] Q. Chen, H. Ghenniwa, and W. Shen. Web-Services Infrastructure for Information Integration in Power Systems. Power Engineering Society General Meeting, 2006. IEEE, 1:11, 2006.

[4] J.O. Dada. Information Exchange Network for the Liberalised Electricity Market with Object-Oriented and Internet-Based Technologies. Fortschritt-Berichte VDI, 2002.

[5] J.O. Dada and H.D Kochs. XML-based open electricity market information exchange network using object-oriented methods. International Journal of Computers and Applications, 27(3):153-160, 2005.

[6] J.O. Dada. Information Exchange Framework for Deregulated Electricity Market in Nigeria. International Journal of Engineering and Technology, 2(6):1052-1061, 2012.

[7] K.M.S. Kumar, A.S. Das, and S. Padmanabhuni. WS-I Basic Profile: a practitioner's view. Proceedings. IEEE International Conference on Web Services, 2004., 2004.

[8] J. Zhu. Web services provide the power to integrate. IEEE Power and Energy Magazine, 1(6):40-49, November 2003.

[9] M.U.Tariq, S. Grijalva, and M. Wolf. Towards a Distributed, Service-Oriented Control Infrastructure for Smart Grid. In Proceedings of the 2011 IEEE/ACM Second International Conference on Cyber-Physical Systems, ICCPS '11, pages 35-44, Washington, DC, USA, 2011. IEEE Computer Society.

[10] Q. Morante, N. Ranaldo, and E. Zimeo. Web Services Workflow for Power System Security Assessment. In 2005 IEEE International Conference on e-Technology, e-Commerce and $e$-Service, pages 374-380. IEEE, 2005.

[11] A. Eberhard and K.N. Gratwick. Light inside: the experience of independent power projects in Nigeria, www.gsb.uct.ac.za/files/Lightinsidev2.pdf. Technical report, The Infrastructure Consortium for Africa, 2012.

[12] Grid-Code: The Grid Code for Nigeria Transmission Systems. Technical report, Nigeria Electricity Regulatory Commission. Available from: http://www.nercng.org/index.php/documentlibrary/func-startdown/27/, 2005.

[13] Distribution-Code: The Distribution Code for Nigeria Electricity Distribution System. Technical report, Nigeria Electricity Regulatory Commission. Available from: http://www.nercng.org/index.php/document-library/funcstartdown/26/, 2005.

[14] Market-Rules: The Market Rules. Technical report, Nigeria Electricity Regulatory Commission. Available from: http://www.nercng.org/index.php/document-library/funcstartdown/127/, 2005.

[15] T. Erl. Service-Oriented Architecture: Concepts, Technology, and Design. Prentice Hall, 2005.

[16] M.P. PAPAZOGLOU, P. TRAVERSO, S. DUSTDAR, and F. LEYMANN. SERVICE-ORIENTED COMPUTING: A RESEARCH ROADMAP. International Journal of Cooperative Information Systems, 17(02):223-255, June 2008.

[17] H. Ghenniwa. Web-services infrastructure for information integration in power systems. In 2006 IEEE Power Engineering Society General Meeting, page 8 pp. IEEE, 2006.
[18] A. Vaccaro, M. Popov, D. Villacci, and V. Terzija. An Integrated Framework for Smart Microgrids Modeling, Monitoring, Control, Communication, and Verification. Proceedings of the IEEE, 99(1):119-132, 2011.

[19] J.O. Dada and P. Mendes. Design and Architecture of Web Services for Simulation of Biochemical Systems. In DILS, pages 182-195, 2009.

[20] D. Jordan and A. Alves. Web Services Business Process Execution Language Version 2 . 0. Language, 11:1-264, 2007.

[21] S. Wang, W. Shen, and Q. Hao. An agent-based Web service workflow model for inter-enterprise collaboration. Expert Systems with Applications, 31(4):787-799, November 2006.

[22] C. Schittko. Web Service Orchestration with BPEL. In Available from: http://citeseerx.ist.psu.edu/viewdoc/download? doi=10.1.1.107.2531\&rep=repl \&type $=p d f, 2003$.

[23] Apache Software Foundation. Apache ODE, Available from: www.apache.org, 2010.

[24] S.T. Lee. The EPRI common information model for operation and planning. In Proc. IEEE Power Engineering Society Summer Meeting, volume 2, pages 866-871, 1999.

[25] A. De Vos, S.E. Widergren, and J. Zhu. XML for CIM model exchange. In Proc. Innovative Computing for Power-Electric Energy Meets the Market. 22nd IEEE Power Engineering Society Int. Conf. Power Industry Computer Applications PICA, pages 31-37, 2001.

[26] X. Wang, N.N. Schulz, and S.Neumann. CIM extensions to electrical distribution and CIM XML for the IEEE radial test feeders. IEEE Transactions on Power Systems, 18(3):10211028, 2003.

[27] J. Xing, H. Yang, and W. Chen. Power System Model Based on CIM and its Application in AVC System Using a CIM Toolkit. In Proc. Asia-Pacific Power and Energy Engineering Conf. (APPEEC), pages 1-4, 2010.

[28] Y. Pradeep, J. Thomas, C.L. Sabari, V.S.K.M Balijepalli, S.R. Narasimhan, and S.A. Khaparde. Towards usage of CIM in Indian Power Sector. In Proc. IEEE Power and Energy Society General Meeting, pages 1-7, 2011.

[29] X. Wang and B.C. Chiu. CIM modeling for Market Management Systems. In Proc. IEEE Power and Energy Society General Meeting, pages 1-5, 2010.

[30] E. Haq, D. Haller, K.A. Rahman, and B. Iverson. Use of Common Information Model (CIM) in Electricity Market at California ISO. In Proc. IEEE Power and Energy Society General Meeting, pages 1-6, 2011.

[31] K. Ballinger, D. Ehnebuske, C. Ferris, M. Gudgin, C.K. Liu, and M. Nottingham. Web Service Interoperability Basic Profile Version 1.1. Available from: http://www.wsi.org/Profiles/BasicProfile-1.1.html. Technical report, 2003. 